\title{
The microstructure and linear superelasticity of cold-drawn TiNi alloy
}

\author{
Y.F. Zheng *, B.M. Huang, J.X. Zhang, L.C. Zhao \\ School of Materials Science and Engineering, PO Box 433, Harbin Institute of Technology, Harbin 150001, People's Republic of China
}

Received 22 February 1999; received in revised form 27 September 1999

\begin{abstract}
The microstructure and linear superelasticity of cold-drawn TiNi alloy have been investigated using CTEM, HREM and in-situ transition electron microscopy observations and tensile tests. The microstructural evolution procedure of the martensite variants with increasing degree of area reduction was found to be that the quantities of (11) Type I, (001) compound, (011) Type I and (111) Type I twinning plates increase gradually with decreasing the amount of the 〈011〉 Type II twinning bands. The corresponding dominant deformation mechanism changed from the coalescence of martensite variants to the reorientation of substructural bands inside martensite variants utilizing the most favorably oriented twin systems. The intrinsic reason for the linear superelasticity of the moderately cold-drawn TiNi alloy was confirmed to be associated with the appearance and disappearance of (001) microtwinning inside the 〈011〉 Type II substructural bands. C 2000 Elsevier Science S.A. All rights reserved.
\end{abstract}

Keywords: Microstructure; Linear superelasticity; TiNi alloy; Martensite

\section{Introduction}

The study of deformation modes in martensite is important as a basis for understanding the shape memory effect associated with the thermoelastic martensitic transformation. Generally speaking, most of the martensites in thermoelastic alloys have twins as a result of the lattice invariant shear or self-accommodation. It is usually observed in thermoelastic alloys that twin boundaries are mobile under stress, and this motion constitutes deformation modes in the martensite concerned. For binary TiNi alloy, the twinning morphologies and modes in the thermal martensite were extensively studied by conventional transmission electron microscopy (TEM) [1-5]. The morphologies of deformed martensite in TiNi alloys after cold working or deformation had been involved [6-11], but the small scale of the laths, the micro-orientation between nominally identical variants and the occurrence of the intersecting variants make any crystallographic structure

\footnotetext{
* Corresponding author. Tel.: + 86-451-6412-163; fax: + 86-4516413-922.

E-mail address: yfzheng@public.hr.hl.cn (Y.F. Zheng)
}

determination by conventional selected area diffraction very difficult. There existed a discrepancy in the dominant twinning modes in the cold worked martensite. Lin et al. [6] suggested that no twinning mode changed in the deformed martensite; Madangopal and Rajarshi [7] reported that (011) Type I twin related parallel plates were the typical microstructure of the TiNi alloy cold worked to $30 \%$ deformation in the martensitic condition. Koike et al. [8] found a small volume fraction of amorphous phase and $\langle 011\rangle$ Type II twin existing in the $30 \%$ thickness reduced TiNi alloy. Further investigation is clearly desirable. Tadaki and Wayman [9] have examined the superelasticity and microstructure in TiNi alloy cold rolled up to about $30 \%$ reduction in thickness and observed the aligned lamellar structure with fine segments along different directions inside each lamellae, and suggested that these subtwins are related to the linear superelasticity. However, until now, the mechanism for linear superelasticity is still unclear, especially short of evidence in the dynamic state.

The purpose of the present study is to investigate systematically the microstructural features of TiNi alloy with the various degrees of the area reduction, of 
particular interest is the dominant twinning modes appearing in the martensite variants at different stages. Based on this, the moderately cold-drawn NiTi alloy is further studied by in-situ TEM observation in tension to understand the nature of the linear superelastic behavior exhibited by the alloy under this microstructural condition.

\section{Experimental details}

A Ti-49.8at.\%Ni alloy was prepared by melting electrolytic nickel and sponge titanium in an induction furnace. The ingot was hot swaged and rolled at $850^{\circ} \mathrm{C}$ to rods with approximately $8 \mathrm{~mm}$ diameter. After annealing at $850^{\circ} \mathrm{C}$ for $1 \mathrm{~h}$, some specimens were colddrawn at room temperature to the extents of $5,8,12$, $14,16,22$ and $30 \%$ reduction in area, respectively. The tensile tests were carried out on an Instron-1186 testing machine with a strain rate of $4.1 \times 10^{-4} \mathrm{~s}^{-1}$. Foil specimens were prepared by twin-jet electropolishing with an electrolyte consisting of $20 \% \mathrm{H}_{2} \mathrm{SO}_{4}$ and $80 \%$ $\mathrm{CH}_{3} \mathrm{OH}$ by volume. TEM observations were carried out by using a Philips CM-12 electron microscope operated at $120 \mathrm{kV}$, equipped with a conventional double tilting stage, and a JEOL 2000EX electron microscope operated at $200 \mathrm{kV}$, equipped with a tensile stage. Selected area and micro-diffraction experiments were used to determine the twinning mode. The beam size in the latter was about $40 \mathrm{~nm}$ in diameter. HREM observations were performed by a JEOL-2000EX electron microscope operated at $200 \mathrm{kV}$ using a top-entry type double-tilt specimen stage with angular ranges of $\pm 10^{\circ}$.

\section{Results}

\subsection{Evolution of the cold-drawn microstructure with area reduction}

As is well known for the as-annealed and undeformed TiNi alloy, the martensite variants exhibit selfaccommodation morphology, with the various twinning boundaries being straight and well defined [1-5,12]. The substructure of each single martensite variant had been confirmed to be dominantly $\langle 011\rangle$ Type II twin [3-5], with a few (111) Type I twin observed [5]. The
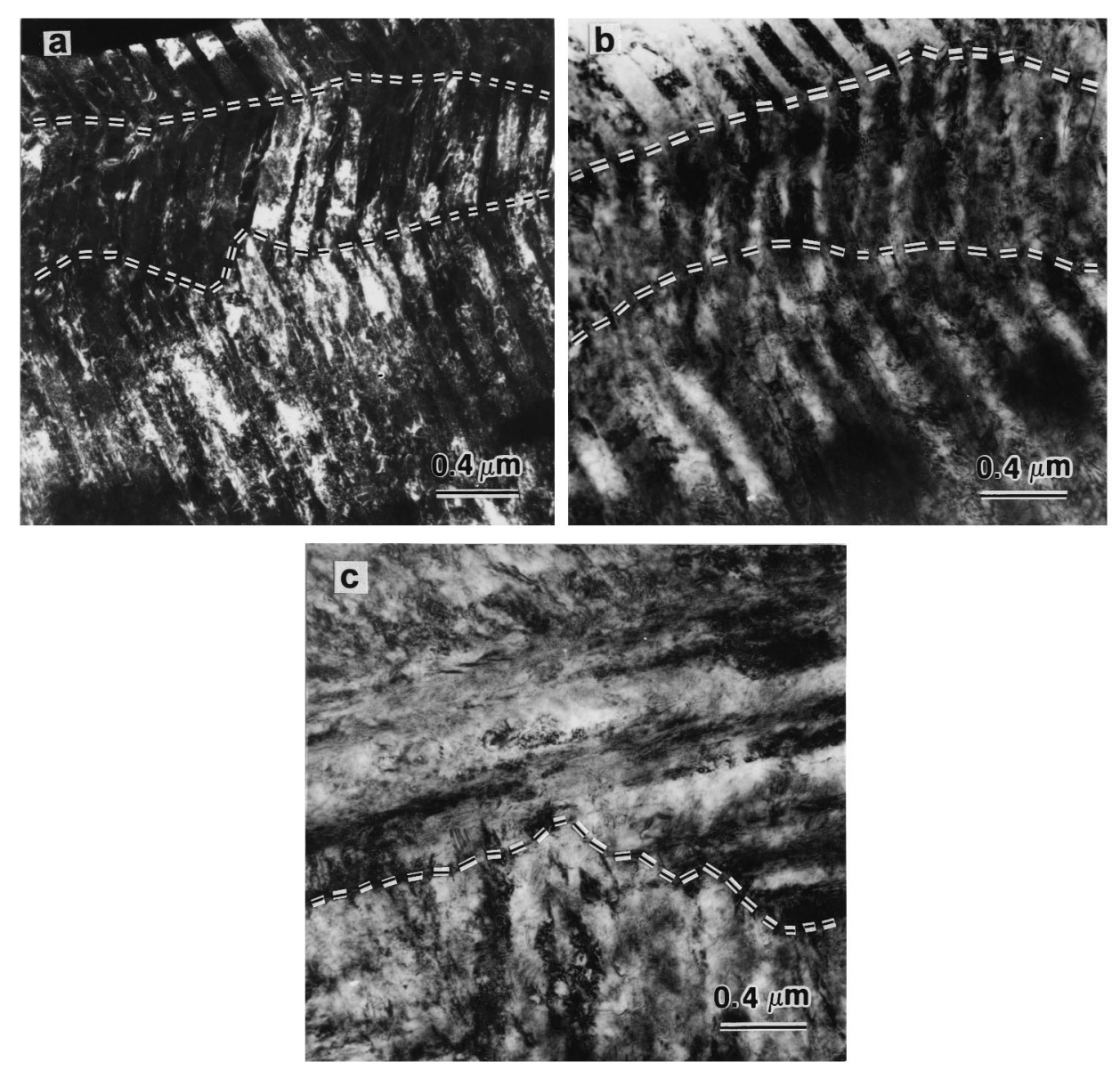

Fig. 1. Typical bright-field images of the deformed martensite variants in the specimens subjected to (a) 5 ; (b) 5; and (c) $8 \%$ area reductions. 
present TEM observations indicate that the coalescence of martensite variants happens after the specimen is subjected to $5 \%$ area reduction, as shown in Fig. 1. The intervariant boundaries, indicated by the dashed lines in Fig. 1(a,b), become curved and irregular. This suggests that the martensite variants in the favorable stress direction accommodate the deformation strain by consuming the neighboring variants in the unfavorable stress direction during the slightly cold-drawn deformation stage. Meanwhile, the width of the internal $\langle 011\rangle$ Type II twinning substructural bands inside each martensite variant shows no obvious change. When the area reduction reaches $8 \%$, both the intervariant boundary and the substructural boundary of the martensite variants become confused and blurred, as shown in Fig. 1(c). Electron diffraction results indicate that the substructure of martensite variant is still dominantly the $\langle 011\rangle$ Type II twinning.

With the increment of the area reduction to $12 \%$, the coalescence of the martensite variants seems difficult to continue because the unfavorable variants have been completely consumed. Fig. 2 shows the typical morphology of the microstructure in the specimen at this deformation stage. The orientations of variants $\mathrm{A}$ and $\mathrm{B}$ are almost identical in Fig. 2(a). The original variant sandwiched between them is wholly consumed and becomes invisible, as clearly shown in the high magnification image Fig. 2(c). Fig. 2(b), the corresponding EDPs taken from area A in Fig. 2(a), indicates that the substructure inside the variant A is the $\langle 011\rangle$ Type II twinning. One noteworthy item is the streak along the $[001]_{\mathrm{M}}$ reciprocal direction, which indicates that a few (001) stacking faults are formed inside the substructural bands. The enlarged HREM image of the area marked by an arrow in Fig. 2(c) is illustrated in Fig. 3, showing the existence of faultings on the (001) plane. Fig. 2(d) depicts more clearly the alternating morphology of many coalesced martensite variants with very small orientation differences. The EDPs derived from areas $\mathrm{C}$ and $\mathrm{D}$ in Fig. 2 (d) are shown in Fig. 2(e,f), respectively. The diffraction pattern in Fig. 2(f) consist of three sets of reflections. The spots derived from the $\mathrm{M}$ and the $\mathrm{T} 1$ are in mirror symmetry of each other with respect to the $(11 \overline{1})$ plane, whereas the spots derived from the M and T2 are consistent with Fig. 2(c) in the work of Knowles and Smith [3], in which the $\langle 011\rangle$ Type II twinning was first reported in the martensite in TiNi alloy. It could be conjectured that no considerable macroscopic strain could be available through variant coalescence, the further deformation procedure would take place mainly inside the martensite variants and the further rearrangement of substructural bands would occur, especially between the $\langle 011\rangle$ Type II and the (11) Type I twinning plates.

With the further increase of the area reduction to $16 \%$, the substructural bands partially adjust their ori- entation by the rearrangement of their internal twinning bands; at the same time, some needle-like plates, as the subunit smaller than the $\langle 011\rangle$ Type II twin related unit, appear obviously inside some substructural bands of martensite variants. Fig. 4 shows the typical morphologies in the specimen under this stage, in which Fig. 4(a,e) show the typical morphological features of the newly generated twinning subunits. Each substructural band has been divided into several segments by these small 'fill-in' structures. The newly formed martensite plates are found to be (001) compound twin related with the matrix part from which they developed, as indicated by the corresponding micro-diffraction patterns (Fig. 4(b,f)). The formation of these microtwins is obviously due to the existence of immobile intervariant boundaries. These small needle-like structures most likely originate from the pre-existing (001) stacking faults and develop outward under the external stress, they become wider and terminate at the next substructural boundary or even penetrate into the neighboring band. From Fig. 4(c), it can also noticed that the conjunct near-identical oriented martensite variants begin to merge into each other. The (11) Type I twinning plates are found to adapt the further colddrawn deformation condition better than the $\langle 011\rangle$ Type II twinning bands, since the number of observations for (11) Type I twinning mode, as listed in Table 1 , increases. For example, it could be noticed in Fig. 4(c) that several martensite plates had rectified their orientation to the direction parallel to the plane B.

Fig. 5 shows the HREM image of the (001) compound subtwinning interface in the $16 \%$ cold-drawn specimen. It can be seen that the interface is relatively straight with one atomic-layer height step existing at the boundary. Sinclair interpreted it in terms of local atomic arrangements associated with faults at the boundary [13]. The strait area marked by arrows in the middle of Fig. 5 exhibits black contrast, which indicates the presence of local violent stress field inside the substructural band.

The appearance of secondary subtwinning plates inside the substructure of martensite variants become more obvious in the specimen subjected to $22 \%$ area reduction, as shown in Fig. 6. On the one hand, the (11) Type I twinning bands inside different martensite variants gradually merge into each other and replace the neighboring $\langle 011\rangle$ Type II twinning bands with the violence of the cold-drawn deformation, as confirmed by Fig. 6(a,b); On the other hand, several kinds of microtwinning structures are found to form inside the substructural bands. Fig. 6(c),(d) depict the newlyformed (011) Type I twinning plates inside a coalesced substructural band, whereas Fig. 6(e,f) suggest a large number of (001) compound twinning plates had been nucleated and grown up inside the favorable oriented substructural bands. 

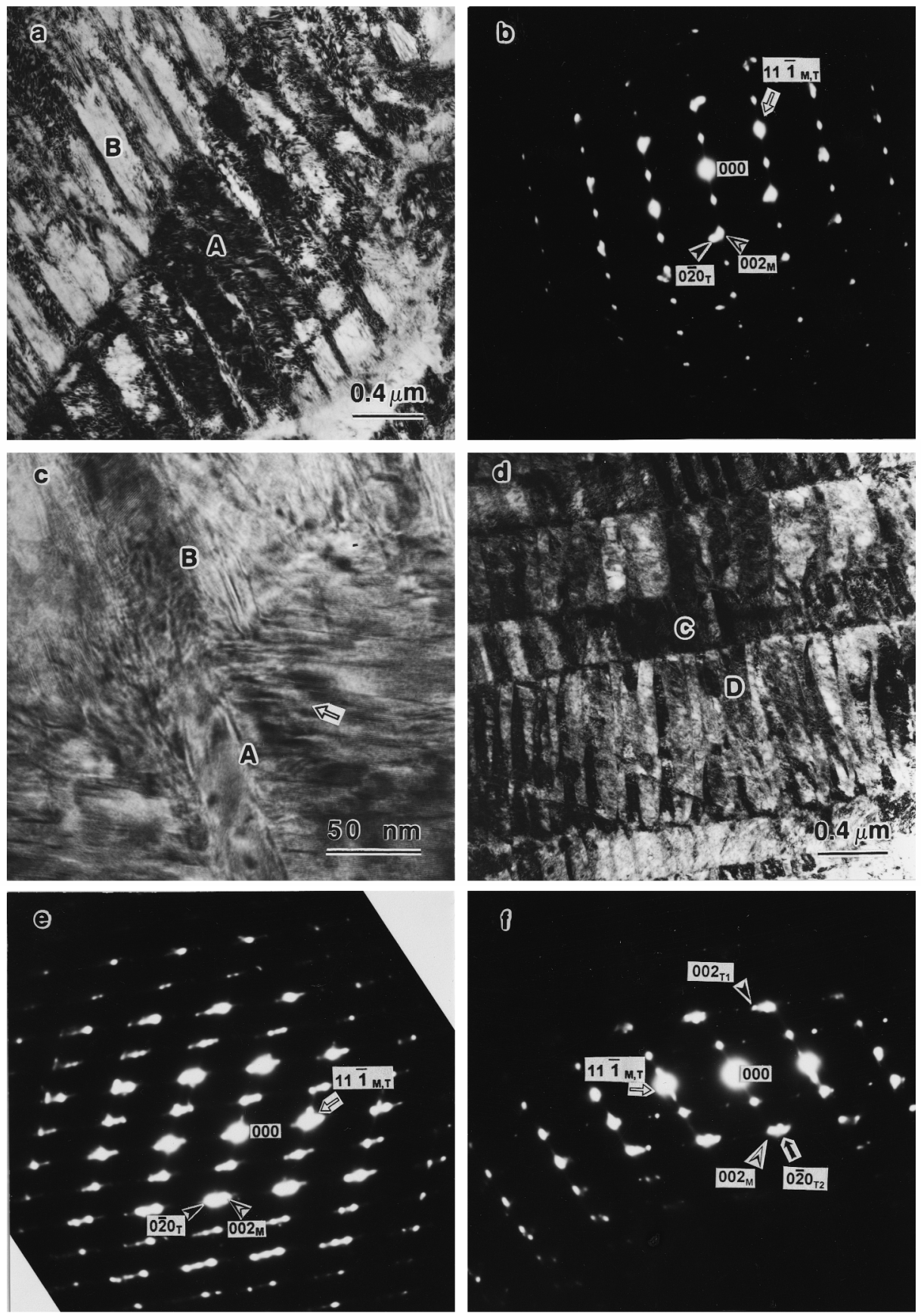

Fig. 2. (a) Typical bright-field image of the deformed martensite variants. (b) EDPs taken from area $A$ in (a), electron beam $/ /[\overline{1} 10]_{\mathrm{M}} / /[101]_{\mathrm{T}}$. (c) Enlarged image of the area near A in (a). (d) Another typical electron micrograph of the deformed martensite variants. (e) EDPs taken from area

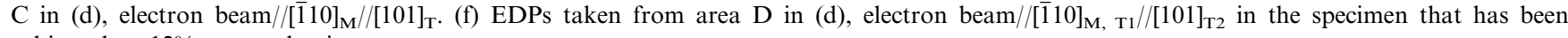
subjected to $12 \%$ area reduction.

When the area reduction is about $30 \%$, the gradually curved martensite substructural bands with different orientations become the dominant microstructural feature inside the specimen. It is difficult to find the original intervariant boundaries, which indicates that the substructural bands completely merged into each other. The substructural bands adjust strongly their orientation through internal twinning to accommodate the certain stress condition at the special position. Many secondary microtwinnings could be easily found inside the individual substructural band, as exemplarily indicated by A and B in Fig. 7(a,b). The separated unit inside the heavily cold-drawn martensite substructural band with a single orientation is very fine, as could be revealed directly by the high-magnification images of Fig. 7(c,e). This has been characterized as "nanocrys- 
tals' by Koike et al. [8]. Electron diffraction analyses indicate that the frequently observed twinning relationship between the neighboring substructural bands is (11) Type I mode, with a small amount of $\langle 011\rangle$ Type II mode still retained. The internal secondary deformation microtwinning inside the substructural bands is found to be (001) compound, (011) Type I and (111) Type I modes. We do not reproduce the former two modes, which have been reported in the figures since the microstructural features are similar. Fig. 7(c,d) show simply the newly-formed (111) Type I twinning plate, whereas Fig. 7(e,f) illustrate the co-existed morphology and the corresponding EDPs of (111) Type I and (111) Type I twinning plates.

\subsection{In situ TEM observation of the tensile deformation behavior of cold-drawn martensite}

Fig. 8 shows the stress-strain curves of the $16 \%$ cold-drawn specimen tensioned to $3.5 \%$ at $20^{\circ} \mathrm{C}$ under different cycling numbers. It could be seen that the complete linear superelasticity is achieved after five cycles. The specimen is cut from the tensile sample after seven cycling deformations. In-situ TEM observation in tension has been carried out to illustrate the variation of the microstructure under different deformation conditions, as shown in Fig. 9. The TEM micrograph after unloading is omitted since it is almost identical to Fig. 9(a). The microstructural change during loading and unloading sequentially can be deduced as follows. Before tension, few 'fill-in' plates could be found inside the $\langle 011\rangle$ Type II twinning substructure, as shown by Fig. 9(a,b). Under the action of the applied stress, new planar twinning plates nucleate and grow up inside the

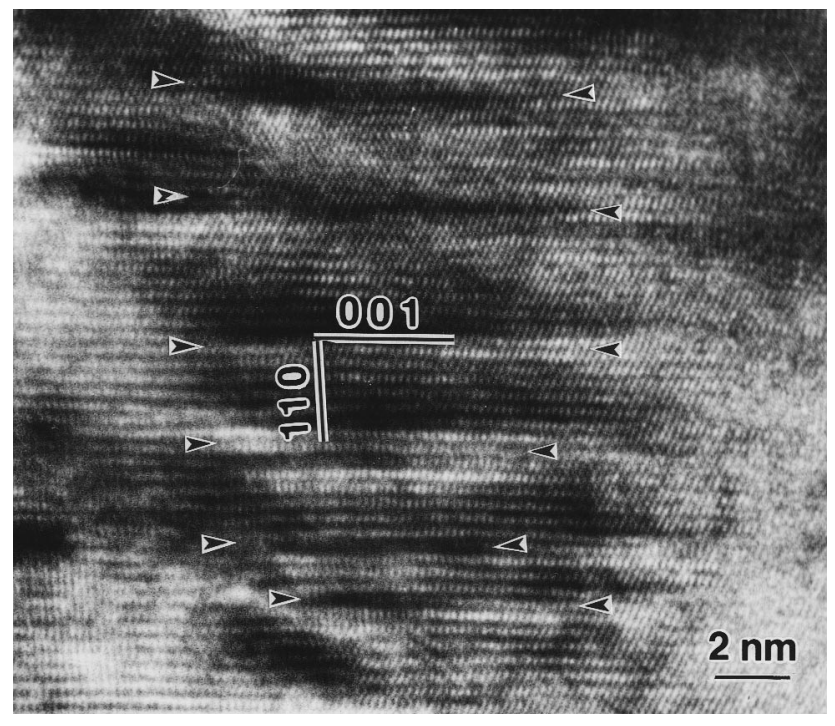

Fig. 3. Enlarged HREM image of the area marked by an arrow in Fig. 2(c) showing faulting on the (001) plane in the $12 \%$ cold-drawn specimen, Electron beam $/ /[\overline{1} 10]_{\mathrm{M}, \mathrm{T}}$. substructural bands in the favorable orientation, then these newly generated twinning strips broaden, associated with the formation of some other new thin plates with the increase of the applied stress, as shown by Fig. 9(c-e). Fig. 9(f), the corresponding diffraction pattern taken from the newly-formed martensite plates, indicates the twinning relationship is (001) compound type; During the unloading, these needle-like (001) twinning martensite plates shrink back and the whole observed area returns to its initial condition, mostly when the load is released. These results suggest that the unusual superelastic behavior of the moderately cold-drawn specimen is caused by the reversible motion of twin boundaries, which associates with the appearance and disappearance of (001) deformation microtwins upon loading and unloading, respectively. The formation of the microtwins is attributed to the fineness of the lamellar martensite crystals due to cold-drawing and the existence of the immobile boundaries between the lamellae, as suggested by Tadaki and Wayman [9].

\section{Discussion}

In the present study, five kinds of twinning modes are observed. It is apparent that the microstructural aspect and the corresponding orientation relationships are determined by the complex stress state at the special position. The whole microstructural evolution procedure for the present initially martensitic TiNi alloy undergoing severe cold-drawing deformation could be rationalized as follows. At the beginning, the coalescence of the martensite variants happens, resulting in curved and distorted intervariant boundaries. After consuming completely the unfavorably oriented martensite variants, the orientation adjustment of the pre-existed substructural bands inside the individual variant occurs. The substructural bands rearrange their orientation by coalescing the disadvantageously oriented bands; at the same time, the development of secondary microtwinnings inside some substructural bands proceeds along with ordinary slip. The secondary microtwinning should be those which counteract the stress field at the band interior most effectively. A different stress field might induce the generation of different kind of microtwinning plates. Detailed conditions for each twinning mode will now be discussed separately.

1. 〈011〉 Type II mode. The substructure of martensite variant at the slight cold-drawn deformation stage is found to be the $\langle 011\rangle$ Type II twinning dominantly. Yet, with the increase of the area reduction, the amount of the $\langle 011\rangle$ Type II twinning gradually reduces. As is well known, the $\langle 011\rangle$ Type II twinning is the most frequently observed twinning mode in the thermal martensite [5], which has also been 

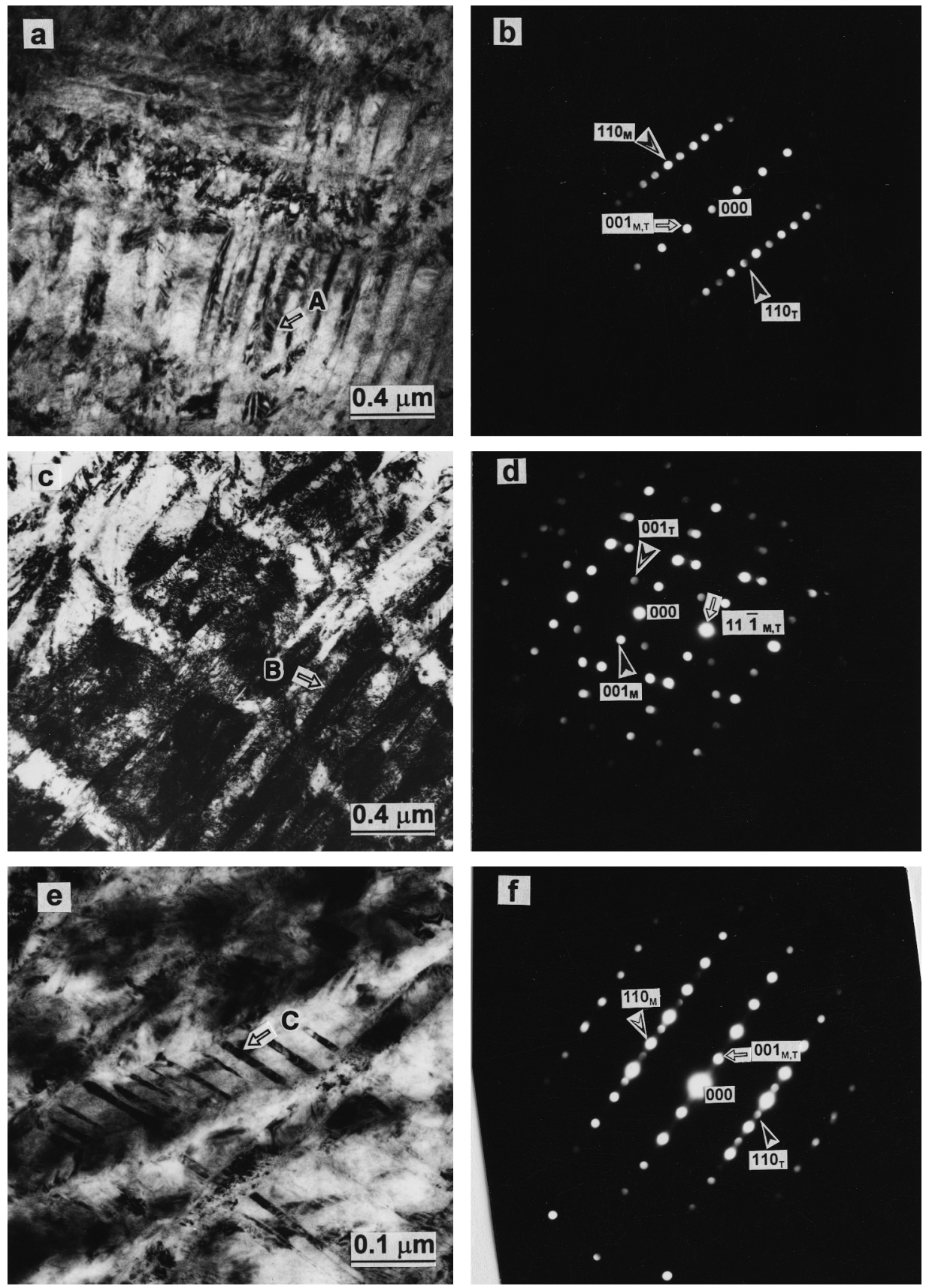

Fig. 4. (a) Typical bright-field image of the deformed martensite variants. (b) Micro-diffraction patterns taken from area A in (a), electron beam $/ /[\overline{1} 10]_{\mathrm{M}, \mathrm{T}}$. (c) Another typical electron micrograph of the deformed martensite variants. (d) Micro-diffraction patterns corresponding to area $\mathrm{B}$ in (c), electron beam $/ /[\overline{1} 10]_{\mathrm{M}, \mathrm{T}}$. (e) High-magnification bright-field image showing the newly formed (001) microtwinning plates. (f) Micro-diffraction patterns derived from area $\mathrm{C}$ in (e), electron beam//[1110 $]_{\mathrm{M}, \mathrm{T}}$ in the specimen subjected to $16 \%$ area reduction.

pointed out theoretically to be a lattice invariant shear [3,4]. Since the microstructure is wholly martensitic at the room temperature for the present alloy before cold-drawing, the appearance of the $\langle 011\rangle$ Type II twinning bands is clearly to provide the necessary lattice invariant shear during the martensitic transformation which had finished before cold-drawing. This kind of twinning orientation seems unfavorable to accommodate the cold-draw- ing deformation strain from the beginning. No obvious change in morphological aspect at the slightly cold-drawn deformation stage is simply due to the intervariant boundary showing more easy migration under the action of the external stress.

2. (11) Type I mode. With the increase of the area reduction amount, the probability of random observation of (111) Type I twinning mode in the present deformed specimens becomes high. In the thermal 
Table 1

Frequency of random observations for different twinning modes in the specimens with different area reductions for the present alloy

\begin{tabular}{|c|c|c|c|c|c|c|}
\hline \multirow[t]{2}{*}{ Amount of cold-drawing ( $\%)$} & \multicolumn{6}{|c|}{ Observation number } \\
\hline & $\langle 011\rangle$ Type II & (111) Type I & (001) compound & (011) Type I & (111) Type I & Total number \\
\hline 5 & 27 & 4 & - & - & - & 31 \\
\hline 8 & 25 & 3 & 1 & - & - & 29 \\
\hline 12 & 23 & 4 & 5 & - & - & 32 \\
\hline 16 & 14 & 8 & 10 & 2 & - & 34 \\
\hline 22 & 6 & 12 & 13 & 2 & - & 33 \\
\hline 30 & 3 & 13 & 10 & 4 & 7 & 37 \\
\hline
\end{tabular}

martensite, the (11) Type I twin was mainly believed to be a deformation twin or a variant accommodation twin [5], which implies that it could accommodate the external stress field more easily, especially the shear strains between martensite variants. A recent study [14] on the TiNi alloy due to tension-compression cyclic deformation showed

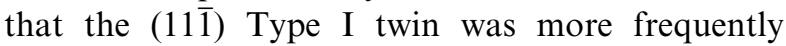
observed in the cycled samples. This may be due to the fact that the value of the twinning shear for the (111) Type I twin, 0.3096, is the largest for all the twinning systems observed so far in the B19' martensite [4], as suggested by Xie et al. [14]. A similar situation had also been reported in the TiN$\mathrm{iNb}$ alloy, that the (111) Type I twin was preferentially formed during the stress-induced martensitic transformation [15]. Based on these results, the (111) Type I twinning mode is believed to be the most favorable oriented twinning system in the present martensite and to play a role in deformation twinning.

3. (001) Compound mode. With the violence of colddrawing deformation, more and more (001) compound twinning plates appear inside the present deformed martensite variants. The (001) compound twin is considered to be a deformation twin in the TiNi martensite [5]. As is well known, the slip system [100](001) was suggested to be the most possible in the martensite in TiNi alloy [16]. According to Kudoh et al. [16], a (001) twin is created by slip of $a / 2$ on the (001) plane because of the presence of a partial pseudo-mirror, therefore the (001) compound twin is easily formed. In a previous study on an aged $\mathrm{Ti}-51$ at.\%Ni alloy, the (001) compound twin was found to be the dominant defect in the martensite, where the internal stress fields at the coherent boundary between the fine precipitates and parent phase, and the constraint for the atomic arrangement of the parent phase because of the coherent precipitates are considered to act mutually to introduce its formation [17]. Nishida et al. [5] also reported that the (001) compound twinning striations were found at the area where the elastic strain was heavily accumulated. These previous results agree that the formation of (001) compound twinning is sensitive to the internal stress field and can relieve the elastic interaction inside the martensite variant effectively, which might well explain the present in-situ observation results. Our TEM observation shows that the intervariant boundaries become partially immobile in the moderately cold-drawn specimen subjected to variant coalescence. It might cause local stress concentration near the boundaries, and the stress would gradually pile up and transfer towards the substructural band interior during loading. The generated stress field could be counteracted through twinning on the (001) plane, since it is the most direct and easiest way. However, it leaves the lattice improperly ordered and thus in a high energy state and unstable. When the stress is removed, the microtwin would shrink or disappear in order to return to the initial condition with preferred lattice ordering, unless the reverse way for the microtwin is hindered by unconquerable factors like local overdeformation. The

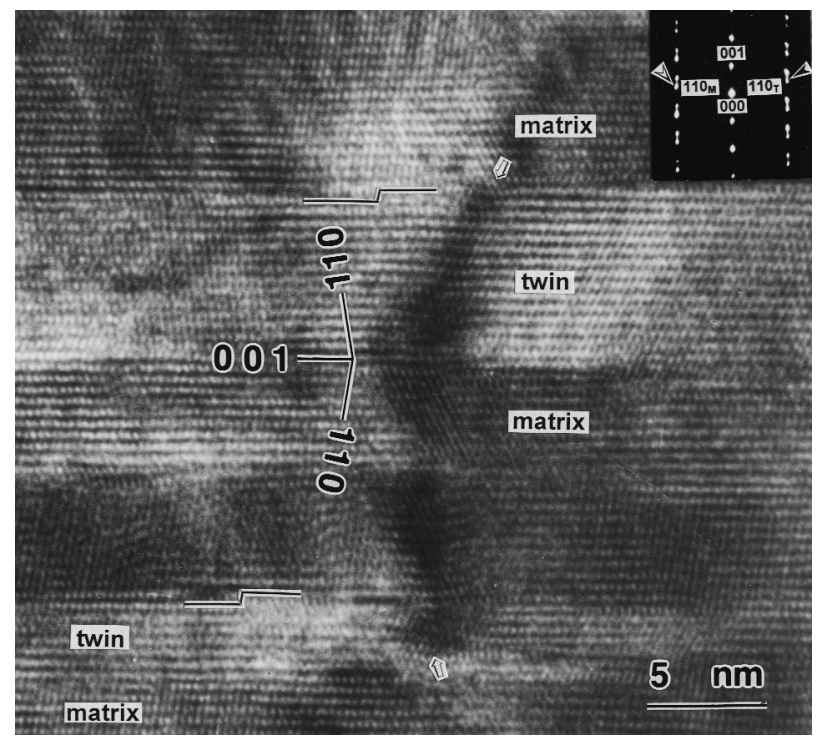

Fig. 5. Enlarged HREM image of the (001) microtwinning boundary in the $16 \%$ cold-drawn specimen, Electron beam $/ /[\overline{1} 10]_{\mathrm{M}, \mathrm{T}}$. 

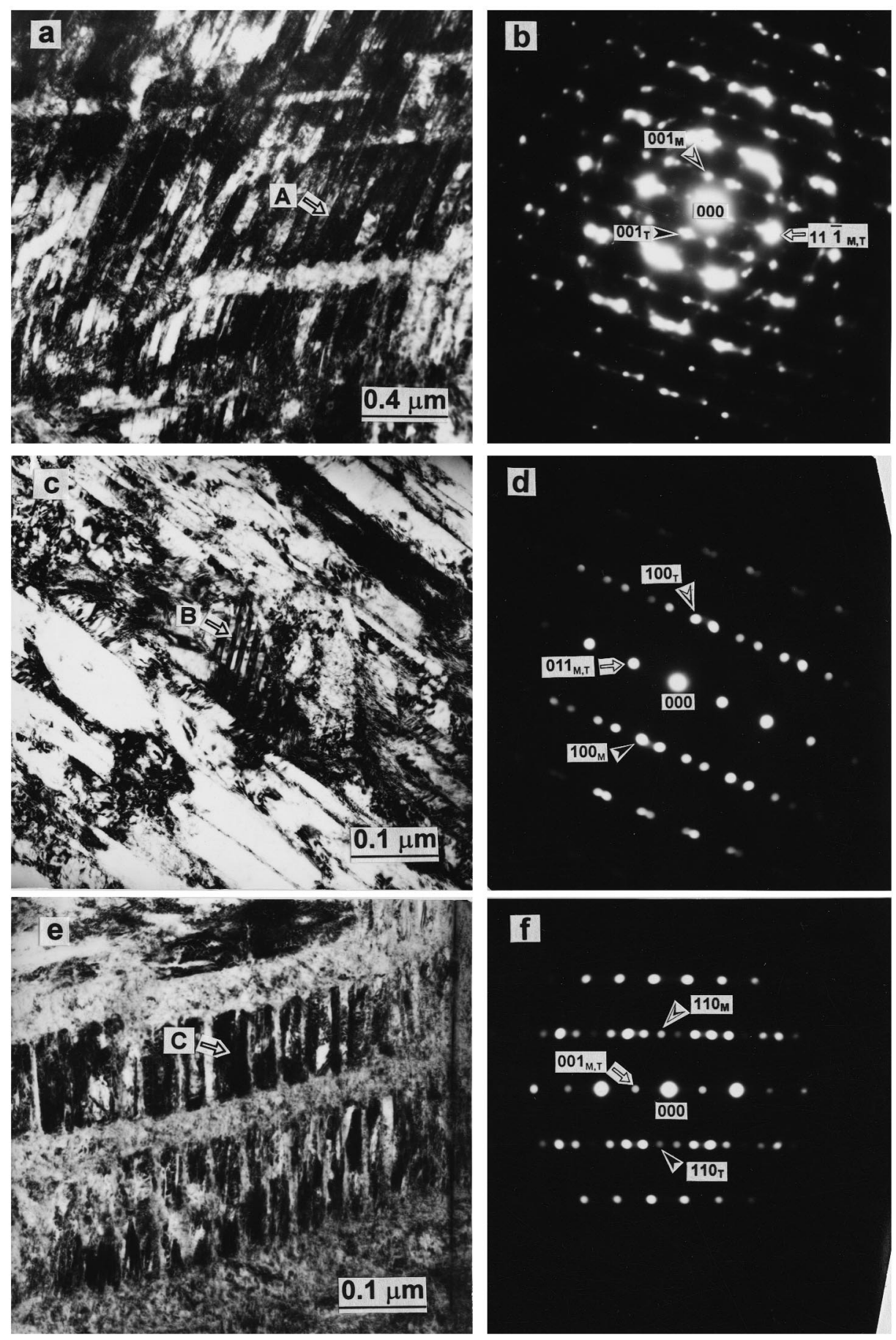

Fig. 6. (a) Typical bright-field image of the deformed martensite variants. (b) EDPs taken from area $\mathrm{A}$ in (a), electron beam//[1 10$]_{\mathrm{M}, \mathrm{T}}$. (c) High-magnification electron micrograph showing the newly generated (011) Type I twinning plates. (d) Micro-diffraction patterns corresponding to area $\mathrm{B}$ in (c), electron beam $/ /[01 \overline{1}]_{\mathrm{M}, \mathrm{T}}$. (e) High-magnification bright-field image showing the newly formed (001) twinning plates. (f) Micro-diffraction patterns derived from area $\mathrm{C}$ in (e), electron beam//[110 $]_{\mathrm{M}, \mathrm{T}}$ in the specimen subjected to $22 \%$ area reduction.

driving force for the reverse motion of the microtwinning boundaries may be supplied by the repulsive force between twinning dislocations themselves [18] or between the twinning dislocations and the dislocations at the intervariant boundaries that constitute the barriers for further variant conversion [9]. In other words, it may be related to reducing the tapered portion of the microtwinning plates, or due to residual stresses, a desire for accommodation dislocations in the twin boundaries to maintain an even spacing [19].

4. (011) Type I mode. In the present study, the (011) Type I twinning plates are found to be formed in the moderately and severely cold-drawn specimens. 
This was suggested to be a lattice invariant shear rather than a sort of a deformation twin in the thermal martensite in TiNi alloy [5]. The microstructural aspect of the (011) Type I twins in the present study is the alternating platelets with regular spacing, which shows the typical feature of the lattice invariant shear. This is in agreement with the viewpoint of Madangopal and Rajarshi [7], who suggested the (011) Type I twinning lamella was derived from a single martensite plate in which the (011) plane was only the intervariant interface or transformed from $\langle 011\rangle$ Type II twin assisted by slip.

5. (111) Type I mode. The (111) Type I twinning plate is found in the present severely cold-drawn specimen. It was first reported in the tensioned $\mathrm{TiNi}$ alloy by the single-crystal X-ray diffraction method [16], and as yet has not been confirmed to exist in the thermal martensite in TiNi alloy by the TEM
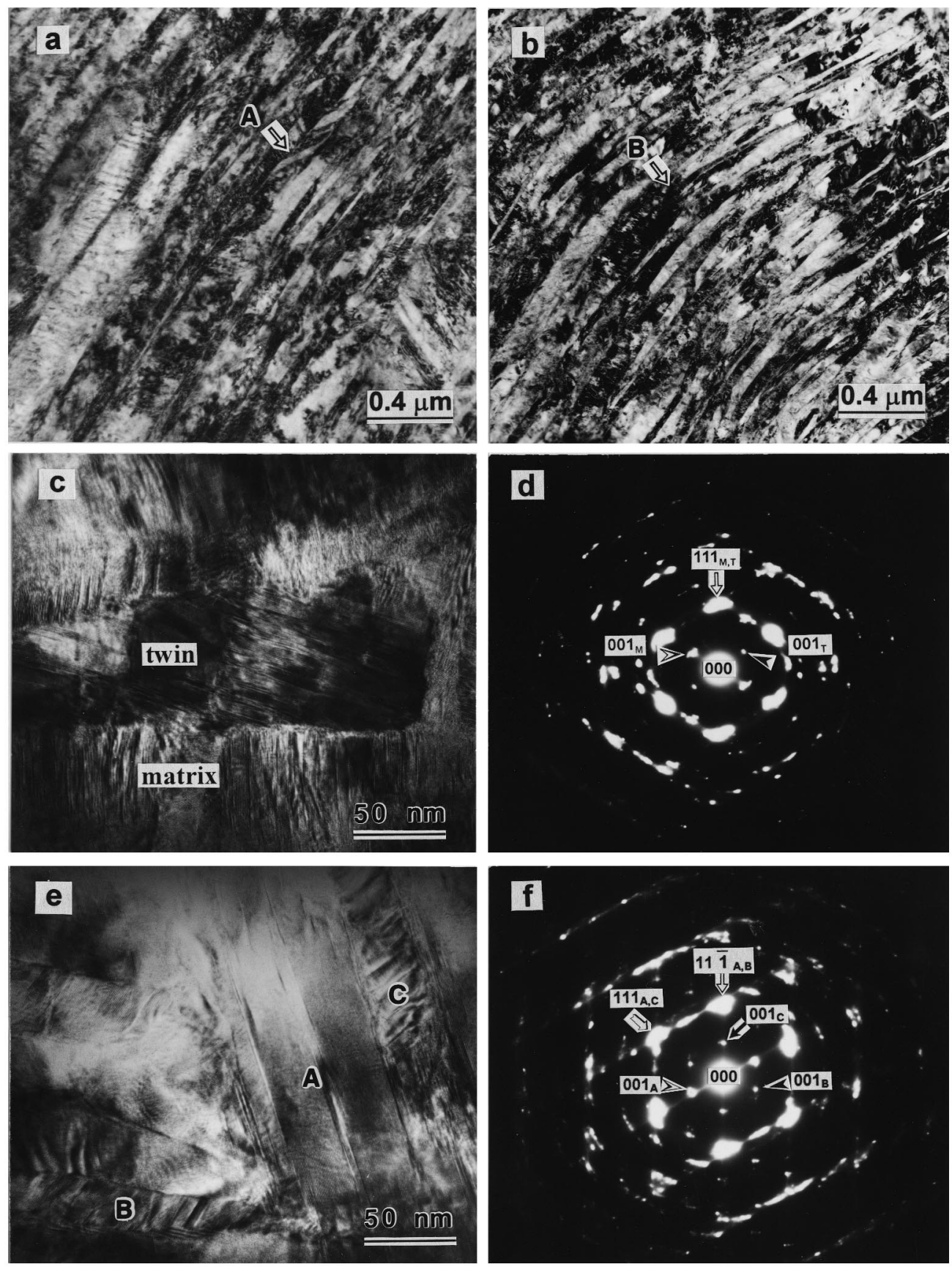

Fig. 7. (a) and (b) Typical bright-field images of the deformed martensite variants. (c) High-magnification electron micrograph showing the newly generated (111) Type I twinning plates. (d) EDPs corresponded to the whole area in (c), electron beam//[1 110 $]_{\mathrm{M}, \mathrm{T}}$. (e) High-magnification bright-field image illustrating the coexisting twinning plates. (f) EDPs derived from the whole area in (e), electron beam//[110 $]_{M, T}$ in the specimen subjected to $30 \%$ area reduction. 


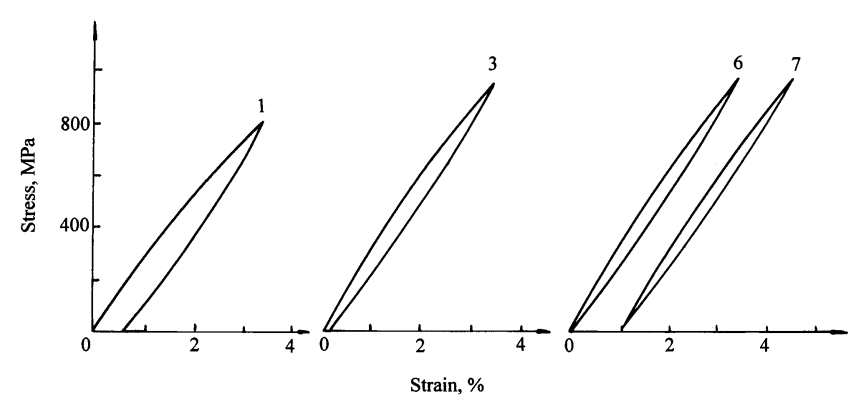

Fig. 8. Stress-strain curves of the $14 \%$ cold-drawn specimen tensioned to $3.5 \%$ at $20^{\circ} \mathrm{C}$.

technique [5]. Recently, it was found in the TiNi alloy due to tension-compression cycle deformation [14] and the stress-induced martensite in $\mathrm{TiNiNb}$

alloy [15], respectively. This twinning mode is clearly introduced by cold-drawn deformation; therefore, it should be regarded as deformation twinning.

\section{Conclusions}

The deformation morphologies, mechanism and linear superelasticity of cold-drawn TiNi alloy have been revealed through CTEM, HREM and in-situ TEM observations, and tensile tests. The results are summarized as follows.

1. When the area reduction is less than $12 \%$, the coalescence of martensite variants happens mainly with little change of the internal $\langle 011\rangle$ Type II twinning substructure, resulting in the curved and distorted intervariant boundaries.
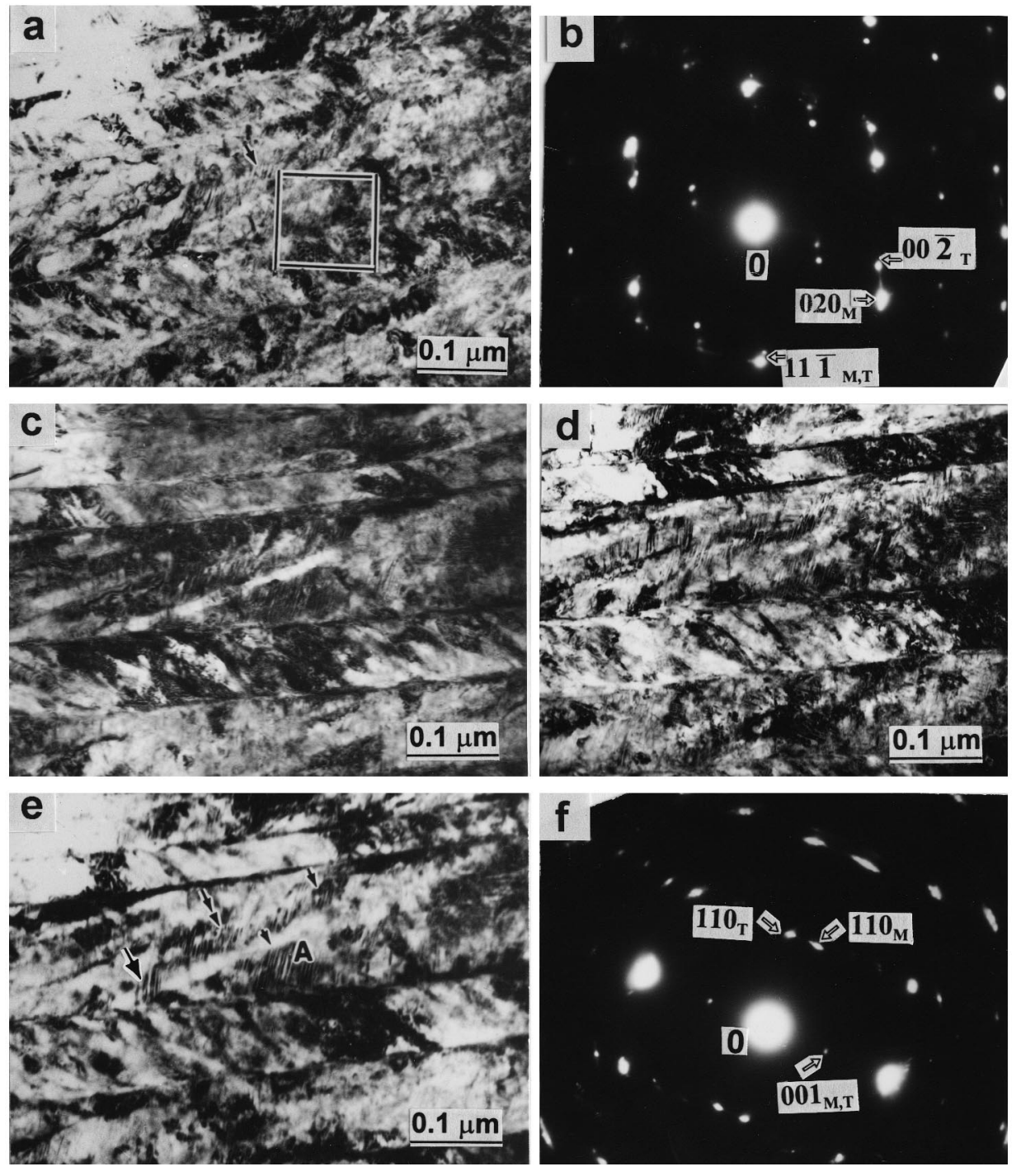

Fig. 9. TEM in-situ observations showing the microstructural changes with the applied stress in the specimen subjected to $14 \%$ area reduction at $20^{\circ} \mathrm{C}$. (a) Bright-field image before tensile deformation. (b) EDPs taken from the framed area in (a), electron beam//[110 $]_{\mathrm{M}} / /[101]_{\mathrm{T}}$. (c) Bright-field image corresponding to a strain of approximately $0.8 \%$. (d) Bright-field image corresponding to a strain of approximately $1.8 \%$. (e). Bright-field image corresponding to a strain of approximately $2.5 \%$. (f) EDPs derived from area A in (e), electron beam//[110 $]_{\mathrm{M}, \mathrm{T}}$. 
2. When the area reduction is higher than $16 \%$, the adjustment and development of the internal twinnings inside individual martensite variant becomes the dominant deformation mode. Many (11) Type I, (001) compound, (011) Type I and (111) Type I twinning plates are gradually formed and coalesced by consuming the pre-existing $\langle 011\rangle$ Type II twinning bands. The orientations of deformed martensite plates become diverse and the size of the smallest martensite unit with a certain orientation becomes smaller.

3. The microscopic mechanism for the linear superelasticity of the moderately cold-drawn TiNi alloy is confirmed to be due to the appearance and disappearance of (001) deformation microtwins upon loading and unloading.

\section{Acknowledgements}

Project 59471029 supported by the National Natural Science Foundation of China.

\section{References}

[1] K. Otsuka, T. Sawamura, K. Shimizu, Phys. Status Solidi (a) 5 (1971) 457.
[2] S.P. Gupta, A.A. Johnson, Trans. Jpn. Inst. Metals 14 (1973) 292.

[3] K.M. Knowles, D.A. Smith, Acta Metall. 29 (1981) 101.

[4] T. Onda, Y. Bando, T. Ohba, K. Otsuka, Mater. Trans. JIM 33 (1992) 354.

[5] M. Nishida, K. Yamauchi, I. Itai, H. Ohgi, A. Chiba, Acta Metall. Mater. 43 (1995) 1219.

[6] H.C. Lin, S.K. Wu, T.S. Chou, H.P. Kao, Acta Metall. Mater. 39 (1991) 2069.

[7] K. Madangopal, B. Rajarshi, Scr. Metall. Mater. 27 (1992) 1627.

[8] J. Koike, D.M. Parkin, M. Nastasi, J. Mater. Res. 5 (1990) 1414.

[9] T. Tadaki, C.M. Wayman, Scr. Metall. 14 (1980) 911.

[10] K.N. Melton, O. Mercier, Metall. Trans. 9A (1978) 1487.

[11] H.A. Mohamed, T. Washburn, J. Mater. Sci. 12 (1977) 469.

[12] M. Nishida, K. Yamauchi, I. Itai, H. Ohgi, A. Chiba, Acta Metall. Mater. 43 (1995) 1229.

[13] R. Sinclair, Am. Inst. Phys. Conf. Proc. 53 (1979) 269.

[14] Z. Xie, Y. Liu, J.V. Humbeeck, Acta Mater. 46 (1998) 1989.

[15] L.C. Zhao, W. Cai, in: K. Inoue, K. Mukherjee, K. Otsuka, H. Chen (Eds.), Proceedings of Displacive Phase Transitions and Their Applications in Materials Engineering, TMS, 1998, p. 93.

[16] Y. Kudoh, M. Tokonami, S. Miyazaki, K. Otsuka, Acta Metall. 33 (1985) 2049.

[17] M. Nishida, C.M. Wayman, A. Chiba, Metallography 21 (1988) 275.

[18] K. Otsuka, H. Sakamoto, K. Shimizu, Scr. Metall. 11 (1977) 41.

[19] T.W. Duerig, G.R. Zadno, Engineering Aspects of Shape Memory Alloys, Butterworth-Heinemann, London, 1990, p. 369. 